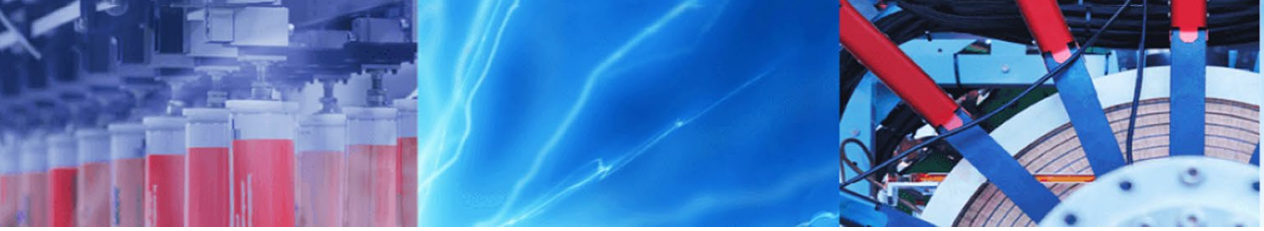

Research Article

\title{
Optimization of grinding efficiency considering surface integrity of bearing raceway
}

\author{
Zhou Chang $^{1} \cdot$ Qian Jia $^{1}$
}

() Springer Nature Switzerland AG 2019

\begin{abstract}
In order to improve the grinding efficiency of bearing raceways, a multi-objective optimization method that considers the surface integrity constraints of the bearing raceway is proposed. Appropriate design points are selected through an orthogonal test, and a response surface model of the grinding parameters along with the response output is established on the basis of the test results. Explicit expressions for the grinding force and roughness are formulated, and the constraints necessary to meet the quality requirements are obtained. The genetic algorithm NSGA-II is applied to the multi-objective optimization of grinding time and material removal rate, and the Pareto set is solved. The results of the study show that using optimized grinding parameters can reduce the grinding time and material removal rate, and can also help broaden the available knowledge base on high-speed and high-efficiency grinding technology.
\end{abstract}

Keywords Surface integrity · Response surface model · Orthogonal test · Multi-objective optimization · Grinding efficiency

\section{Introduction}

The importance of bearing raceways, which form the working surface of bearings, is quite evident. Grinding is widely used in the manufacture of bearings and is one of the most important processing methods used in the fabrication of bearing raceways. An important requirement often encountered in the optimization of such processes is the further improvement in grinding efficiency without compromising on grinding quality.

Surface integrity of the bearing raceway is the most important index used to evaluate their grinding quality [1]. The surface integrity of bearing raceway usually includes two indexes, namely the surface texture and surface physical characteristics. The surface texture or accuracy of the bearing raceway is usually related to the accuracy of bearing rotation, while the surface physical characteristics of the bearing raceway are usually associated with the life of the bearing.
The optimization of grinding process parameters can be regarded as a multi-objective optimization problem [2]. The surface integrity of the bearing raceway can be regarded as a constraint condition, and the grinding efficiency of the bearing can be regarded as an objective function. Generally, two kinds of algorithms, namely evolutionary algorithms and swarm intelligence algorithms, are used to solve multi-objective optimization problems [3].

Evolutionary algorithms generally consist of the genetic algorithm and its improved variants [4-6]. Saravanan [7] uses a genetic algorithm to optimize planegrinding parameters. Compared with the quadratic programming method, the production cost is reduced and the productivity is improved. Sedighi [8] uses the genetic algorithm and neural networks to predict grinding roughness and grinding removal rate. Swarm intelligence algorithms mainly include the ant colony and particle swarm optimization algorithms and their improved variants $[9,10]$. Baskar [11] uses the ant colony algorithm

Zhou Chang, starismyfriend@163.com | 'State Key Laboratory for Manufacturing System Engineering, Xi'an Jiaotong University, Xi'an 710054, Shaanxi, People's Republic of China.

SN Applied Sciences (2019) 1:679| https://doi.org/10.1007/s42452-019-0697-8 
to optimize plane-grinding parameters, reduce production costs, and improve productivity. Lee [12] uses particle swarm optimization to optimize grinding parameters, while ensuring grinding quality, improving productivity, and reducing production costs.

Several useful and insightful studies have been carried out regarding practical problems in grinding optimization, and these serve well to guide engineering applications [13-15].

From the above literature survey, it is evident that studies on the grinding efficiency of bearing raceways, and especially multi-objective optimization studies, are scarce. Many grinding optimization studies do not consider the constraint of bearing raceway surface integrity. In this context, the present study establishes a first-order response surface model of the grinding tangential force and grinding roughness by an orthogonal test, and explicit expressions for the grinding tangential force and roughness are obtained. The genetic algorithm NSGA-II is used to solve the two-objective optimization problem of grinding time and grinding removal rate. The results of this study are expected to prove valuable for practical applications.

\section{Experiment}

Grinding experiments were carried out on a 3 MK 1420 bearing raceway grinder. The grinding wheel material is corundum, and the model is P80 X $10 \mathrm{X} 20 \mathrm{~A} 80 \mathrm{KV} 60$. The grinding wheel is dressed using a single-point diamond pen dresser. An emulsion is used as the grinding fluid, and the grinding workpiece is the outer ring of an angularcontact ball bearing (model: 7014AC) after heat treatment. The outer diameter of the ring specimen is $110 \mathrm{~mm}$, inner diameter is $98 \mathrm{~mm}$, width is $20 \mathrm{~mm}$, and width of the raceway is $7 \mathrm{~mm}$. The material of the ring is bearing steel GCr15. The processing equipment is shown in Fig. 1.

Plunge grinding process is used to machine the bearing raceway. Plunge grinding is a grinding method in which there is no axial motion but only radial motion between grinding wheel and workpiece. The grinding process of the bearing raceway can be divided into three stages: rough grinding, fine grinding, and spark out. When all the three stages are completed, the grinding wheel is dressed using a diamond pen. It can thus be assumed that the characteristics of the grinding wheel are the same before the grinding of each bearing raceway.

In Fig. 2, $U_{1}$ represents the feed speed of rough grinding and $U_{2}$ is the feed speed of fine grinding. The feed speed of spark out, $U_{3}$, is equal to $0 . t_{1}$ is the rough grinding time, $t_{2}$ is the fine grinding time, and $t_{3}$ is the spark out time.

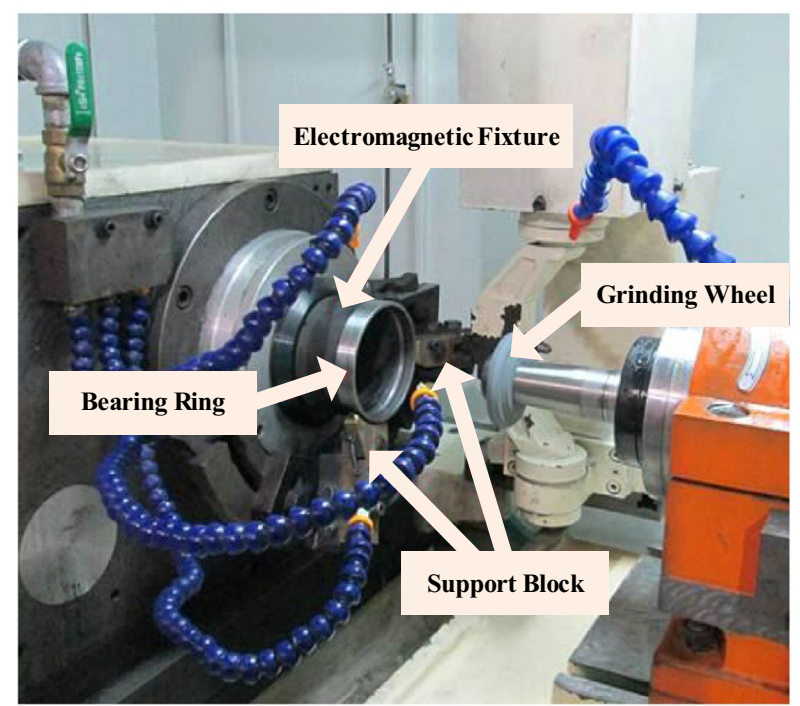

Fig. 1 Grinding equipment

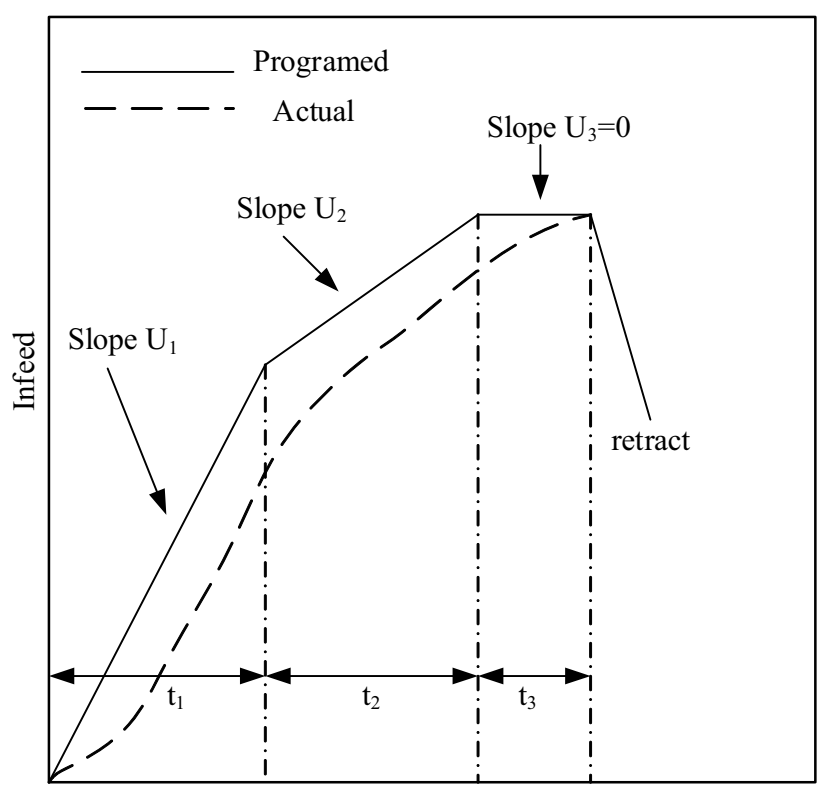

Time

Fig. 2 Grinding time

\section{Optimization algorithm}

An important requirement in the manufacture of bearings is the improvement in bearing accuracy, while maintaining the required grinding quality. This requirement can be expressed as a multi-objective optimization problem that is described mathematically as follows: 


$$
\left.\begin{array}{l}
x=\left[x_{1}, x_{2}, \ldots x_{r}\right]^{\top} \\
\min f_{i}(x)=\left\{f_{1}(x), f_{2}(x), \ldots, f_{s}(x)\right\} \\
\text { s.t. } g_{j}(x) \leq 0 j=1,2, \ldots, p
\end{array}\right\} .
$$

In the given problem formulation represented as the set of Eq. (1), $X$ is the variable to be optimized, $r$ is the number of variables, $f_{i}(x)$ is the optimization objective function, $s$ is the number of objective functions, $g_{j}(x)$ is the constraint function, and $p$ is the number of constraint functions.

As per the problem defined in this study, improvement in the grinding efficiency can be achieved by reducing the grinding time and improving the material removal rate.

\subsection{Selection of objective function}

In the plunge grinding of bearing raceway, a shorter grinding time is considered desirable. First, the total grinding time is considered as the optimization objective function denoted by,

$T=t_{1}+t_{2}+t_{3}+t_{4}$.

where $t_{1}$ is rough grinding time, $t_{2}$ is fine grinding time, $t_{3}$ is spark out time, and $t_{4}$ is dressing time. In order to ensure that the grinding parameters remain similar for each grinding operation, the grinding wheel is dressed once after grinding each bearing raceway. The dressing time of the grinding wheel is fixed. Hence, only the time durations of the rough grinding, fine grinding, and spark out phases can possibly be optimized.

Now, the modified grinding time $T^{\prime}$ can be written as the optimization objective function, as follows:

$\min T^{\prime}=t_{1}+t_{2}+t_{3}$.

$t_{1}=\frac{a_{\mathrm{pr}}}{u_{1}}$.

$t_{2}=\frac{a_{\mathrm{pf}}}{u_{2}}$

$a_{\mathrm{pr}}$ is the depth of rough grinding, $U_{1}$ is the feed speed of rough grinding, $a_{\mathrm{pf}}$ is the depth of fine grinding, and $U_{2}$ is the feed speed of fine grinding.

The total grinding depth of bearing raceway can be divided into rough grinding depth and fine grinding depth. The total grinding depth is determined by the machining allowance, which is $250 \mu \mathrm{m}$.

$a_{\mathrm{pr}}+a_{\mathrm{pf}}=250$.

Now, the material removal rate $Z$ is considered as the second objective function.

$Z=b v_{\mathrm{w}} a_{\mathrm{pf}}$. $b$ is grinding width, $v_{\mathrm{w}}$ is work piece speed, and $a_{\mathrm{pf}}$ is grinding depth.

According to the function theory, the maximum value of $f(x)$ is equivalent to the minimum value of $-f(x)$. For the convenience of optimization, the function $Z^{\prime}$ may be considered as the objective function of optimization, as follows:

$\min Z^{\prime}=-b v_{\mathrm{w}} a_{\mathrm{pf}}$.

\subsection{Variable constraints}

In order to optimize the grinding time and material removal rate, some intermediate variables are eliminated, and six appropriate variables mentioned below are selected for the optimization.

$X=\left[v_{s}, v_{w}, a_{\mathrm{pf}}, u_{1}, u_{2}, t_{3}\right]$.

where $v_{\mathrm{s}}$ is grinding speed, $v_{\mathrm{w}}$ is workpiece speed, $a_{\mathrm{pf}}$ is grinding depth. $u_{1}$ is the feed speed of rough grinding, $u_{2}$ is the feed speed of fine grinding, and $t_{3}$ is the spark out time.

Due to the limitations of machine tools and grinding wheels, the constraints applicable to these six variables can be represented by the following:

$0 \leq v_{s} \leq 45$.

$0 \leq v_{\mathrm{w}} \leq 1.5$.

$0 \leq a_{\mathrm{pf}} \leq 30$

$5 \leq t_{3} \leq 8$.

$3 \leq u_{1} \leq 10$

$1 \leq u_{2} \leq 3$

\section{Response surface model}

\subsection{Representation of constraints}

Grinding quality refers to the surface integrity of the bearing raceway. The surface integrity of bearing raceway includes the surface texture and surface physical characteristics. In the context of this study, surface texture refers to the roughness of the bearing raceway. The roughness of bearing raceway is regarded as the constraint condition for the multi-objective optimization. In the grinding of bearing raceway, it is considered that a roughness less than $0.3 \mu \mathrm{m}$ is sufficient to meet the grinding quality 
requirements [16]. Thus, the roughness constraint can be formulated as:

$0 \leq R_{a} \leq 0.3$.

There are three indexes pertaining to the surface physical characteristics of bearing raceway, namely the residual stress, hardness, and metallographic structure of the bearing raceway. Rowe [17] studied the effect of grinding temperature on the surface integrity of the bearing raceway: when the grinding temperature is less than $400{ }^{\circ} \mathrm{C}$, the residual stress on the bearing raceway is compressive, hardness is more than $60 \mathrm{HRC}$, and the metallographic structure does not change. Thus, the grinding temperature is regarded as the constraint condition of multi-objective optimization. In the bearing raceway grinding, it is considered that the grinding temperature must be less than $400{ }^{\circ} \mathrm{C}$ to meet the grinding quality requirements [17]. So, the next constraint can be formulated as:

$0 \leq T \leq 400$.

\subsection{Response surface modeling}

In this study, the model of grinding roughness and grinding force is established by the response surface method.

Grinding roughness is assumed to be a function of $v_{s}$, $v_{\mathrm{w}}, a_{\mathrm{pf}}[18]$. In order to facilitate the optimization, the function of grinding roughness is needed.

$R_{a}=f\left(v_{s}, v_{\mathrm{w}}, a_{\mathrm{pf}}\right)$

The grinding temperature can be calculated by the following formula [17]:

$T=q_{\mathrm{w}} \frac{\mathrm{C}}{\beta_{\mathrm{w}}} \sqrt{\frac{l_{\mathrm{c}}}{v_{\mathrm{w}}}}$.

In the formula, $C$ is the coefficient, 1 for shallow grinding, $q_{\mathrm{w}}$ is the heat entering the workpiece, $\beta_{\mathrm{w}}$ is the thermal characteristic, and $I_{c}$ is the real contact arc length.

$q_{\mathrm{w}}=\varepsilon \frac{F_{\mathrm{t}} \cdot v_{\mathrm{s}}}{l_{\mathrm{c}} \cdot b}$.

In the given equation, $F_{\mathrm{t}}$ is tangential force, $\varepsilon$ is heat distribution ratio, and $b$ is grinding width.

It can be seen from the above formula that as long as the tangential grinding force model is obtained, the temperature model can be obtained.

Tangential grinding force is considered as a function of $v_{s^{\prime}} v_{\mathrm{w}^{\prime}}$ and $a_{\mathrm{pf}}[17]$.

$F_{\mathrm{t}}=f\left(v_{\mathrm{s}}, v_{\mathrm{w}}, a_{\mathrm{pf}}\right)$.
In order to facilitate the optimization, we need to obtain the function of the grinding tangential force.

The response surface method is a method of approximating the implicit function with a polynomial explicit function [19]. The response surface model is established. First, the experiment is designed, the data are fitted, and finally the predictions related to non-test points are carried out.

Because there are three variables that affect the grinding force and roughness, this study uses three factors and three levels in the orthogonal test table to carry out experiments.

The first-order model is adopted in the response surface model, and the approximate function can be represented as:

$y=\beta_{0}+\beta_{1} x k+\beta_{2} x_{2}+\beta_{3} x_{3}+\cdots \beta_{k} x_{k}+\varepsilon$.

In Eq. (11), $\beta_{i}$ is determined by the least-square method, and $\varepsilon$ is a random error.

Orthogonal test is a three-level and three-factor test.

In the orthogonal grinding experiment, the tangential grinding force is obtained by measuring the grinding current, and thereby calculating the power. The measuring instrument is the current probe Tek A622. In the orthogonal grinding test, the surface roughness Ra of the bearing raceway was measured using a PGI contact aspheric surface measuring instrument. The measurement results are presented in Table 1.

The tangential force regression analysis was compiled using MATLAB. The significance level was set to 0.02 , and the confidence level was set to $95 \%$. The explicit function expression of the tangential grinding force model is obtained.

$F_{\mathrm{t}}=209.4733 \cdot v_{\mathrm{s}}^{-.04635} v_{\mathrm{w}}^{0.5278} a_{\mathrm{af}}^{0.2473}$.

The roughness regression analysis was performed using MATLAB. The significance level was set to 0.02 , and

Table 1 Measurement results from grinding [20]

\begin{tabular}{llllll}
\hline $\begin{array}{l}\text { Group } \\
\text { num- } \\
\text { ber }\end{array}$ & $\begin{array}{l}\text { Wheel } \\
\text { speed } \\
v_{\mathrm{s}} / \mathrm{m} / \mathrm{s}\end{array}$ & $\begin{array}{l}\text { Work } \\
\text { piece } \\
\text { speed } \\
v_{\mathrm{w}} / \mathrm{m} / \mathrm{s}\end{array}$ & $\begin{array}{l}\text { Grind- } \\
\text { ing } \\
\text { depth } \\
a_{\mathrm{pf}} / \mu \mathrm{m}\end{array}$ & $\begin{array}{l}\text { Grinding } \\
\text { force/N }\end{array}$ & $\begin{array}{l}\text { Roughness/ } \\
\mu \mathrm{m}\end{array}$ \\
\hline 1 & 20.9 & 0.5 & 5 & 55.1 & 0.31 \\
2 & 20.9 & 0.8 & 10 & 81.3 & 0.35 \\
3 & 20.9 & 1.1 & 15 & 107.4 & 0.32 \\
4 & 29.3 & 0.5 & 10 & 50.5 & 0.26 \\
5 & 29.3 & 0.8 & 15 & 73.2 & 0.26 \\
6 & 29.3 & 1.1 & 5 & 66.7 & 0.25 \\
7 & 37.7 & 0.5 & 15 & 55.4 & 0.18 \\
8 & 37.7 & 0.8 & 5 & 52.6 & 0.16 \\
9 & 37.7 & 1.1 & 10 & 75.4 & 0.18 \\
\hline
\end{tabular}


the confidence level was set to $95 \%$. The explicit function expression of the roughness model is obtained as follows:

$$
R_{\mathrm{a}}=7.205 v_{\mathrm{s}}^{-1.0545} v_{\mathrm{w}}^{-0.0027} a_{\mathrm{pf}}^{0.0655} \text {. }
$$

The explicit expressions of grinding roughness and grinding temperature can be obtained using Eqs. (12) and (13), which lay the foundation for the next multiobjective optimization.

\section{Multi-objective optimization}

In this study, the objective function is the minimum grinding time and maximum material removal rate. Grinding quality is regarded as a constraint condition, and the multiobjective optimization problem is established. Genetic algorithm NSGA-II is used for multi-objective optimization. This algorithm, proposed by Deb, is considered to be one of the most effective evolutionary algorithms [21, 22]. NSGA-II is an improvement in the conventional genetic algorithm. First, it designs a fast, non-dominant operator followed by an individual congestion distance operator, and lastly, an elite strategy selection operator [23].

A set of Pareto solutions is obtained by multi-objective optimization design, and the set of solutions cannot optimize all the objective variables optimal. We can select an optimal solution from the Pareto solution set for the optimal solution.

The optimization is performed using MATLAB. In the genetic algorithm NSGA-II, the population is 50, the crossover probability is 0.6 , and the mutation probability is 0.2 .

As can be seen from Fig. 3, the grinding time and material removal rate converge to a stable value after

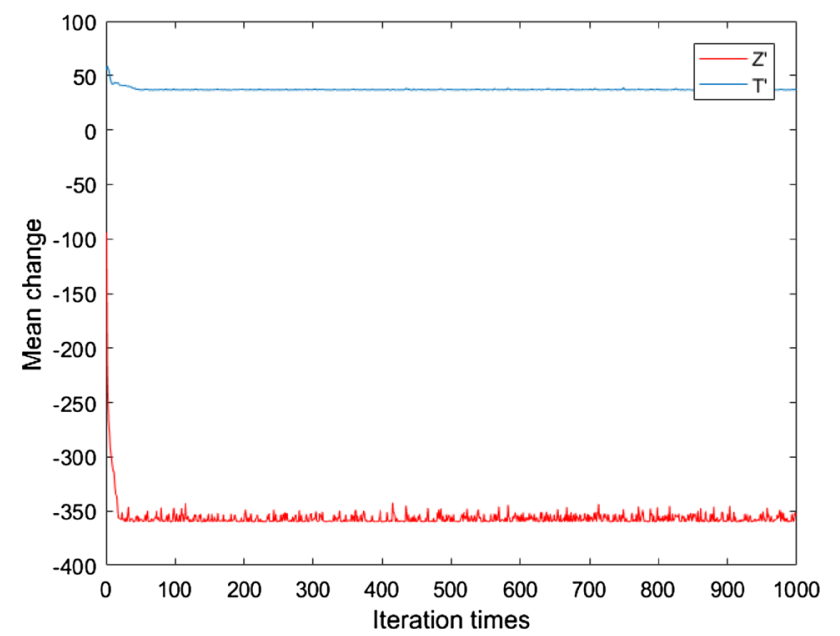

Fig. 3 Optimization iteration

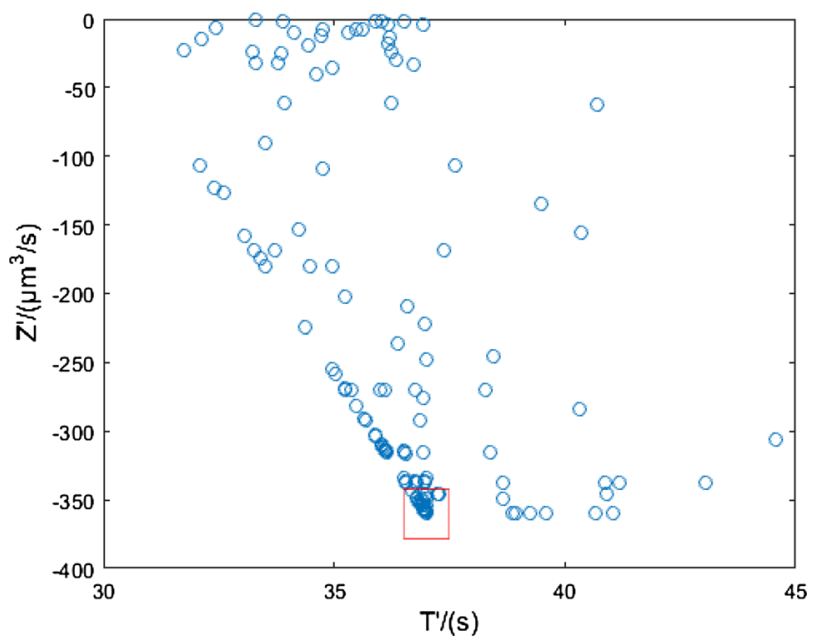

Fig. 4 Pareto set obtained from the analysis

approximately 100 iterations. This shows the high convergence speed of genetic algorithm NSGA-II. It is -360 , which indicates that the maximum material removal rate can reach 360 um 3/s. 37 s, which means that the grinding time can reach $37 \mathrm{~s}$.

As can be seen from Fig. 4, the Pareto front surface is fairly uniform, and the Pareto curve shows a downward trend.

This shows that the genetic algorithm NSGA-II can effectively find the Pareto solution. As can be seen from Fig. 4, the two objective functions are contradictory and follow the polyline relationship. The red box in the figure indicates the optimal solution, which maximizes the grinding removal rate and minimizes the grinding time. Based on the actual grinding process requirements, a few reasonable solutions can be selected. In this way, the combination of grinding parameters will be more flexible.

\section{Comparison of optimization results}

Based on the result of the orthogonal experiment, a set of optimal grinding parameters that satisfy the grinding quality requirements are selected. The optimum grinding parameters are the No. 5 test parameters: $29.3 \mathrm{~m} / \mathrm{s}$ wheel speed, $0.8 \mathrm{~m} / \mathrm{s}$ work piece speed, and 15 um grinding depth. The other grinding parameters are based on those recommended by the bearing manufacturers. The rough grinding speed is $5 \mu \mathrm{m} / \mathrm{s}$ and the finishing speed is $1 \mu \mathrm{m} / \mathrm{s}$ and the polishing time is $7 \mathrm{~s}$. With this group of grinding parameters, the roughness of the grinding surface is $0.26 \mu \mathrm{m}$, and the physical properties of the grinding surface are satisfactory. Empirical method comes from bearing manufacturer. 
Table 2 Comparison of parameters obtained by the empirical and optimization methods

\begin{tabular}{lll}
\hline Design variable & Empirical method & $\begin{array}{l}\text { Optimi- } \\
\text { zation } \\
\text { method }\end{array}$ \\
\hline$v_{\mathrm{s}} / \mathrm{m} / \mathrm{s}$ & 29.3 & 31.3 \\
$v_{\mathrm{w}} / \mathrm{m} / \mathrm{s}$ & 0.8 & 1.5 \\
$a_{\mathrm{pf}} / \mu \mathrm{m}$ & 15 & 30 \\
$u_{1} / \mu \mathrm{m} / \mathrm{s}$ & 5 & 10 \\
$u_{2} / \mu \mathrm{m} / \mathrm{s}$ & 1 & 3 \\
$T_{3} / \mathrm{s}$ & 7 & 5 \\
$T^{\prime} / \mathrm{s}$ & 69 & 37 \\
$Z^{\prime} / \mu \mathrm{m}^{3} / \mathrm{s}$ & -96 & -360 \\
\hline
\end{tabular}

As can be seen from Table 2, in the new optimized grinding parameters, the speed of the grinding wheel increases by only about $6.8 \%$. This shows that the selected grinding wheel speed is reasonable with respect to the grinding parameters of the orthogonal test combined with the empirical method. For the other grinding parameters, the new optimization method has nearly doubled the other grinding parameters. This shows that the combination of the orthogonal experiment and empirical method has shortcomings, and optimization is necessary.

Compared with the empirical method, the grinding time associated with the optimized method is reduced by $46.3 \%$, while the material removal rate increased by $275 \%$. This indicates that the new optimization method has considerable potential engineering applications and can be used in the grinding of bearing raceways.

\section{Conclusion}

(1) The first-order response surface model of the grinding tangential force and grinding roughness was established by orthogonal test. The explicit expressions for the tangential force and roughness are obtained, and the constraint conditions that satisfy the quality requirements are obtained.

(2) Taking the grinding time and material removal rate as objective functions and grinding quality parameters as constraints, a multi-objective optimization problem is proposed. The genetic algorithm NSGAII is used to solve the multi-objective optimization problem, and a Pareto solution set is obtained. The optimal solution is selected from among the members of the Pareto solution set.
(3) Compared with the empirical method, the grinding time is reduced by $46.3 \%$, and the material removal rate is increased by $275 \%$ after optimization. The grinding efficiency has also been greatly improved. This indicates that the method has significant potential for practical engineering applications.

\section{Compliance with ethical standards}

Conflict of interest The authors declare that they have no conflict of interest.

\section{References}

1. Davim JP (2010) Surface integrity in machining, vol 1848828742. Springer, Berlin

2. Deb K (2014) Multi-objective optimization, search methodologies. Springer, Berlin, pp 403-449

3. Deb K, Sindhya K, Hakanen J (2016) Multi-objective optimization, decision sciences: theory and practice. CRC Press, Boca Raton, pp 145-184

4. Zitzler E, Thiele L (1999) Multiobjective evolutionary algorithms: a comparative case study and the strength Pareto approach. IEEE Trans Evol Comput 3(4):257-271

5. Coello CAC, Lamont GB, Van Veldhuizen DA (2007) Evolutionary algorithms for solving multi-objective problems, vol 5. Springer, Berlin

6. Back T (1996) Evolutionary algorithms in theory and practice: evolution strategies, evolutionary programming, genetic algorithms. Oxford University Press, Oxford

7. Saravanan R, Asokan P, Sachidanandam M (2002) A multiobjective genetic algorithm (GA) approach for optimization of surface grinding operations. Int J Mach Tools Manuf 42(12):1327-1334

8. Sedighi $M$, Afshari $D$ (2010) Creep feed grinding optimization by an integrated GA-NN system. J Intell Manuf 21(6):657-663

9. Dorigo M, Birattari M, Blum C et al (2008) Ant colony optimization and swarm intelligence. In: 6th international conference, ANTS 2008, Brussels, Belgium, September 22-24, 2008, proceedings, vol 5217. Springer

10. Bonabeau E, Dorigo M, Theraulaz G (1999) Swarm intelligence: from natural to artificial systems. Oxford University Press, Oxford

11. Baskar N, Saravanan R, Asokan P et al (2004) Ants colony algorithm approach for multi-objective optimisation of surface grinding operations. Int J Adv Manuf Technol 23(5-6):311-317

12. Lee $T$, Ting $T$, Lin $Y$ et al (2007) A particle swarm approach for grinding process optimization analysis. Int J Adv Manuf Technol 33(11):1128-1135

13. Malkin S, Koren Y, Ber A (1980) Off-line grinding optimization with a micro-computer. CIRP Ann Manuf Technol 29(1):213-216

14. Malkin S (1981) Grinding cycle optimization. CIRP Ann Manuf Technol 30(1):223-226

15. Xiao G, Malkin S (1996) On-line optimization for internal plunge grinding. CIRP Ann Manuf Technol 45(1):287-292 
16. Abrāo AM, Aspinwall DK (1996) The surface integrity of turned and ground hardened bearing steel. Wear 196(1):279-284

17. Rowe WB (2013) Principles of modern grinding technology. William Andrew, Norwich

18. Malkin S, Guo C (2008) Grinding technology: theory and application of machining with abrasives. Industrial Press Inc., Norwalk

19. Khuri Al, Mukhopadhyay S (2010) Response surface methodology. Wiley Interdiscip Rev Comput Stat 2(2):128-149

20. Deb K, Agrawal S, Pratap A et al (2000) A fast elitist non-dominated sorting genetic algorithm for multi-objective optimization: NSGA-II. In: International conference on parallel problem solving from nature, pp 849-858

21. Carley KM, Kamneva NY, Reminga J (2004) Response surface methodology. DTIC Doc. https://doi.org/10.1007/978-3-64204898-2_492
22. Deb K, Pratap A, Agarwal S et al (2002) A fast and elitist multiobjective genetic algorithm: NSGA-II. IEEE Trans Evol Comput $6(2): 182-197$

23. Li H, Zhang Q (2009) Multiobjective optimization problems with complicated Pareto sets, MOEA/D and NSGA-II. IEEE Trans Evol Comput 13(2):284-302

Publisher's Note Springer Nature remains neutral with regard to jurisdictional claims in published maps and institutional affiliations. 